\title{
Characterizing Stress-dependent Complex and Relaxation Moduli of Dense Graded Asphalt Mixtures ${ }^{1}$
}

\author{
Linglin $\mathrm{Li}^{\mathrm{a}, \mathrm{b}}$, Wenlong $\mathrm{Li}^{\mathrm{a}}$, Zhongyuan Wang ${ }^{\mathrm{a}}$, Mansheng Dong ${ }^{\mathrm{a}}$, Yuqing Zhang ${ }^{\mathrm{b}}$, \\ ${ }^{a}$ School of Automotive \& Transportation Engineering, Hefei University of Technology, Hefei, China \\ ${ }^{\mathrm{b}}$ School of Engineering and Applied Science, Aston University, Birmingham, U.K. \\ * Corresponding author at: Aston University, Aston Triangle, Birmingham B4 7ET, U.K. \\ y.zhang10@aston.ac.uk (Y. Zhang)
}

\begin{abstract}
The objective of this paper is to investigate the stress-dependence of the complex and relaxation moduli of dense graded asphalt mixtures. Complex modulus tests at four different confining pressures were conducted. Then, the stress dependence of the long-term equilibrium modulus of asphalt mixture based on Prony series model was modeled by Uzan model. The Maxwell components of the relaxation modulus were determined by a two-step method. Finally, the stress dependence of the relaxation modulus was quantified by relaxation spectrum of the material. The results show that for the selected dense-graded asphalt mixtures, the dynamic modulus increases, and phase angle decreases with the increasing confining pressures at relatively low reduced loading frequency. However, the stress dependence is insignificant in a relatively high-reduced loading frequency. The Uzan model coefficient of the long-term equilibrium modulus of the relaxation modulus can be determined effectively by the proposed deviatoric stress master curves. To obtain reliable and accurate Maxwell component coefficients, it is recommended that: a) the measured loss moduli rather than the storage moduli should be used to calculate Maxwell component coefficients if collocation method is utilized, and b) a log-scaled least squared regression minimization objective should be used as the regression objective for the coefficient determination. The relaxation spectrum of asphalt mixture can be modeled by Gaussian function, where the size of the relaxation spectrum increases but the shape and position of the relaxation spectrum remain unchanged when the confining pressure increases.
\end{abstract}

Keywords: Asphalt mixture; Complex modulus; Relaxation modulus; Uzan model; Stress dependence; Master curve

\footnotetext{
1 Accepted manuscript of an article published by Construction and Building Materials. The final publication is available online via: https://doi.org/10.1016/j.conbuildmat.2018.10.162
} 


\section{Introduction}

Considering the frequency and temperature dependence of the asphalt mixture in pavement under repeated traffic loads, dynamic modulus of asphalt mixture is being widely utilized, which correlates well with the field performance of the asphalt pavements $[1,2]$. Now the investigations of the dynamic modulus are mainly focused on the test configurations, conditions [3] and the construction of the dynamic modulus master curve [4,5]. The existing studies also demonstrated that confining pressures could significantly affect the dynamic moduli of asphalt mixtures. Lacroix et al. [6] found that the linear viscoelastic characteristics of asphalt mixtures are affected by the confinements and the elastic modulus at equilibrium is stress dependent. Zeiada et al. [7] found that a confinement of $138 \mathrm{kPa}$ was the threshold value, below which a higher confinement will dramatically increase the dynamic modulus of asphalt mixture. In contrast, an increase of the confining pressure above $138 \mathrm{kPa}$ would insignificantly increase its modulus. Sotil et al. [8] developed point-slope and slope proportion methods to predict the confined dynamic modulus from the unconfined modulus. Pellinen and Witczak [9] presented a method to construct a stress-dependent complex modulus master curve of asphalt mixture based on $k_{1}$ to $k_{3}$ nonlinear elasticity model.

The stress-dependent properties and behaviors of paving materials have been characterized by various models. Witczak and Uzan [10] proposed a universal material model to represent the resilient modulus of the unbound granular materials, which reasonably represents the stiffening effect observed by laboratory experiments. Lytton [11] argued that unbound granular materials in pavements were normally unsaturated and applied the principles of unsaturated soil mechanics to the above model by adding a matric suction term to the bulk stress. Lade and Nelson [12] proposed a nonlinear, isotropic stress dependent model for the elastic behavior of granular materials based on the principle of conservation of energy. Collop et al. [13] developed a stress-dependent elasto-viscoplastic constitutive model with damage for asphalt mixture.

Given the master curves of dynamic modulus and phase angle, the coefficients of the Prony series model can be identified to apply this model to the theoretical and numerical predictions. Many researchers developed different methods to obtain the $E_{i}$ in Prony series model from the 
master curve data. Baumgaertel and Winter [14] proposed a useful technique to calculate a discrete relaxation modulus. The sensitivity of this solution was tested with model data that were free of experimental error. Emri and Tschoegl [15] proposed an iterative computer algorithm to determine $E_{i}$ from given test data, and the main advantage of this algorithm lies in its avoidance of negative values of relaxation moduli that violate physical meanings. Cost and Becker [16] developed a multidata method which can employ all experimental data and result in the reduction of error in the determination of components of relaxation modulus. Schapery [17] introduced an easily applied collocation method to fit the response of finite-element Prony series model to test stress-strain curves. Tschoegl [18] extended Schapery's method to all response functions such as relaxation modulus or retardation compliance in time scale and harmonic responses of storage and loss moduli or compliances in frequency scale.

Some efforts have been devoted to achieving the stress-dependent properties of long-term equilibrium modulus of asphalt mixtures. However, they only consider the effect of the bulk stress but neglect the effect of octahedral stress. Furthermore, due to lacking a simple and robust method to obtain the accurate and reliable enough $E_{i}$ in the Prony Series model of asphalt mixture, there are no studies of stress-dependent properties of $E_{i}$. Therefore, it is uncertain how the stress affects the viscoelastic properties of asphalt mixture when Prony series model is used. The objective of this paper is to investigate the stress dependence of the asphalt mixture under different combinations of deviatoric and confining stresses. The methodology employed in this paper can be illustrated in Figure 1.

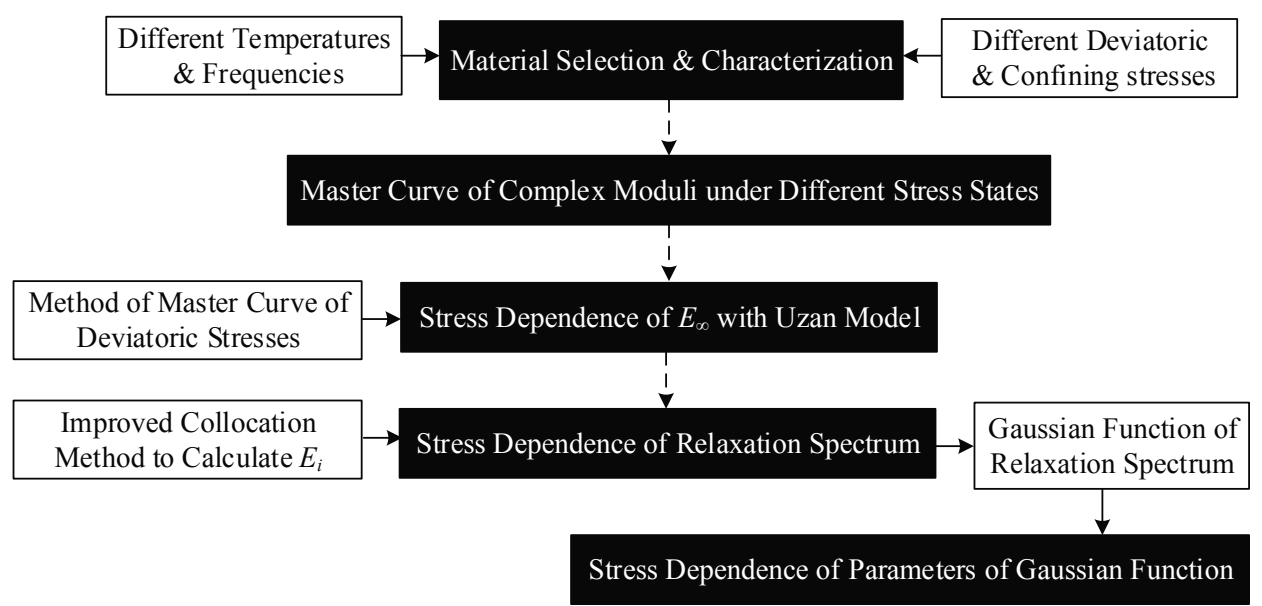

Fig. 1. Research methodology adopted in this study 
The authors understand that the application of the result of this paper into pavement structure is important and some preliminary application of the results can be found elsewhere [19]. In this paper, however, due to the paper length limit, the effects of stress dependent properties on pavement performances were not included but they will be investigated in a following paper, in which the stress dependent viscoelastic parameters will be used to determine the pavement performances under different traffic loading levels with a verified finite element model.

\section{Experiments of Complex Moduli of Asphalt Mixtures under}

\section{Different Confinements}

This section experimentally measured dynamic moduli and phase angles of asphalt mixtures under different confinements. Figure 2 shows the material properties of asphalt binder, aggregates, and mineral powder and the gradation of the aggregates. Cylindrical specimens of the asphalt mixtures were compacted by Superpave Gyratory Compactor to a height of $174 \mathrm{~mm}$ and a diameter of $150 \mathrm{~mm}$. The design air void content (VA) was $4 \%$, and the optimum asphalt content was 4.4\% based on the AASHTO test protocol (i.e., T209 and T166). After demolding, all cylindrical specimens were stored in an environmental chamber for one day, and then cored to $100 \mathrm{~mm}$ in diameter and saw cut to a final height of $150 \mathrm{~mm}$. For each of asphalt mixtures three replicates were tested.

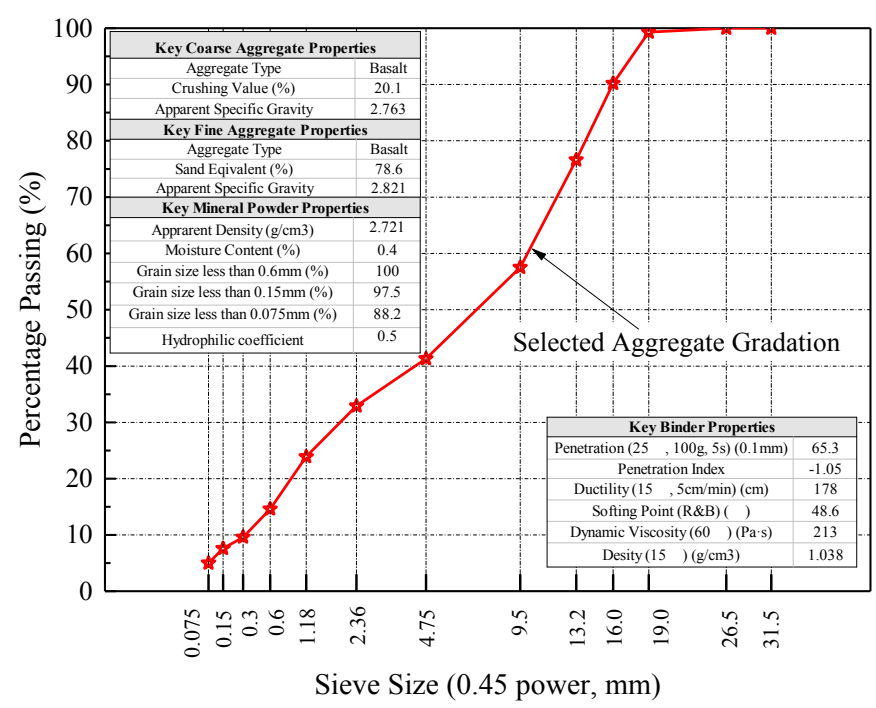

Fig. 2. Key material properties and gradation of aggregates of asphalt mixtures 
The complex modulus tests were conducted at four different confining pressures $(0,35,138$, $207 \mathrm{kPa}$ ) in a strain-controlled manner and in accordance with AASHTO protocol. The key steps of the tests are described as follows. After mounting linear variable differential transducer (LVDT) studs on the lateral side of the specimens, asphalt mixtures were put in the environmental chamber for half day to dry up the glue between the studs and the specimen. Before testing, the specimen was encased in a polyethylene membrane and then put in the chamber at the target testing temperature for at least three hours to ensure that the specimen fully reached the equilibrium temperature. Greased double polyethylene membranes were placed between the specimen ends and the steel loading platens to eliminate the frictional force between them. Three loose-core LVDTs over a gauge length of $70 \mathrm{~mm}$ were glued to the lateral surface of the asphalt mixture specimen at an angle of $120^{\circ}$ apart from each other to record specimen axial deformation. Details of the testing device with the specimen mounted can be found in Figure 3.
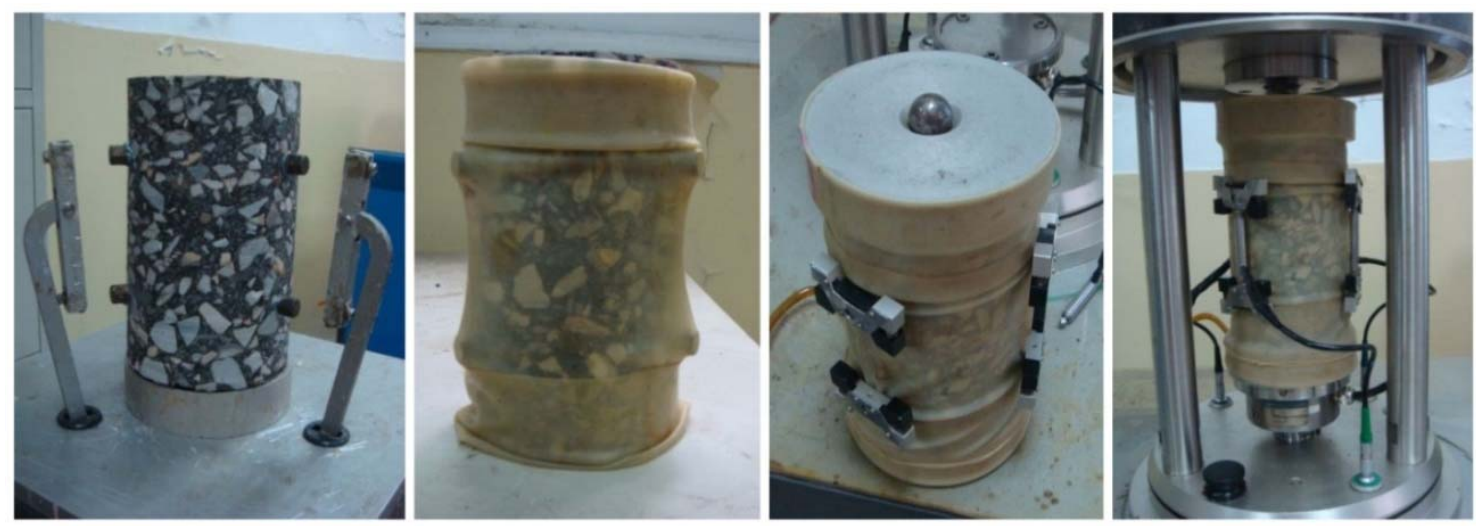

Fig. 3. Details of the experimental setup (Simple Performance Tester)

To minimize any potential damage to asphalt mixture specimen at the low frequency before the next sequential test, tests were conducted in an increasing order of temperature, and at each temperature level, the specimens were tested in a decreasing order of loading frequency. According to Lacroix et al. [6], each of the constant confining pressures $(35,138$, and $207 \mathrm{kPa})$ is applied for at least 1 hour before the confined tests to mitigate the effect of bulk creep. The confinement around the specimen was supplied by pressurized air in the pressure vessel and different combinations of the deviatoric and confinement loads could be applied to the specimen by setting up the software control panel of the Simple Performance Tester for asphalt mixture. 
According to AASHTO Designation T342-11[20], the dynamic load should be adjusted to obtain axial compressive strains between 50 and $150 \mu \varepsilon$. The purpose of this requirement was to ensure that the mixture was tested in an undamaged condition. Although the specific constitutive relationship and its key parameters are considerably affected by asphalt mixture composition and test environment, the viscoplastic strain and damage are always ignored when the axial compressive strain are less than $150 \mu \varepsilon[21,22]$. When the axial compressive strains are below $50 \mu \varepsilon$, the viscoelastic properties of asphalt mixture can be regarded as linear or stress/ strain independent [23]. Nevertheless, the linear viscoelastic characterization is out of the research interest of this paper that is focused on the nonlinear stress-dependent viscoelastic behavior. In consideration of actual strain level used in most dynamic modulus tests, 50 to $150 \mu \varepsilon$ is accepted as the standard controlled strain. Thus, different deviatoric stresses were employed and recorded by setting different axial strain levels ranging from 50 to $150 \mu \varepsilon$. Complex modulus tests are conducted at six frequencies $(0.1 \mathrm{~Hz}, 0.5 \mathrm{~Hz}, 1 \mathrm{~Hz}, 5 \mathrm{~Hz}, 10 \mathrm{~Hz}$, and $25 \mathrm{~Hz})$, four temperatures $\left(10^{\circ} \mathrm{C}, 20^{\circ} \mathrm{C}, 40^{\circ} \mathrm{C}\right.$, and $\left.60^{\circ} \mathrm{C}\right)$, and four confinements $(0,35 \mathrm{kPa}, 138 \mathrm{kPa}$, and $207 \mathrm{kPa}$ ). It should be noted that the negative temperature was not used because the stressdependence of complex and relaxation moduli are negligible at low temperatures when the compressive strain is controlled between 50 and $150 \mu \varepsilon$. At low temperatures, the mixture behaves more linearly with stress independency. The high temperature was selected based on the temperature measurements in the field pavement sections in China, where the highest temperature of surface layer of asphalt pavement is $60^{\circ} \mathrm{C}$ or higher. Therefore, a temperature ranges from 10 to $60^{\circ} \mathrm{C}$ was used to construct the master curve of complex modulus to investigate the nonlinearity of viscoelastic properties of asphalt mixture. For the dynamic modulus, the calculated coefficient of variation (defined as the value of the standard deviation divided by the mean) is between $0.8 \%$ and $17.3 \%$. For the phase angle, the calculated coefficient of variation is between $1.1 \%$ and $20.5 \%$. In general, there is no obvious relationship between the coefficient of variations and test temperatures and frequencies.

\section{Results and Discussion}

\subsection{Stress-dependent Complex Modulus and its Master Curve}


Commonly used master curve models are sigmoidal model for dynamic modulus [4] and beta model for phase angle [24], which can be shown as follows:

$$
\left\{\begin{array}{l}
\log _{10}\left(\left|E^{*}\right|\right)=\delta+\frac{\lambda}{1+\exp \left[\kappa+\gamma \log _{10} \omega_{R}\right]} \\
\varphi=\frac{\varphi_{\max }}{\exp \left\{(1+1 / \beta)\left[\left(\omega_{0} / \omega_{R}\right)^{\beta}-1\right]\right\}\left(\omega_{R} / \omega_{0}\right)^{\beta+1}}
\end{array}\right.
$$

Where $\delta$ and $\lambda$ are the minimum value and the span of $\log _{10}\left(\left|E^{*}\right|\right)$, respectively. $\kappa$ and $\gamma$ are shape parameters. $\varphi_{\max }$ is the maximum value of phase angle. $\omega_{0}$ is the location parameter with dimension of frequency, at which $\varphi_{\max }$ occurs. $\beta$ is the fitting parameter that determines the curvature of the master curve of phase angle. $\omega_{R}$ is reduced frequency that can be obtained by:

$$
\omega_{R}=\alpha(T) \omega
$$

Where $\alpha(T)$ is a time-temperature shift factor. $\omega$ is loading frequency, and $T$ is temperature.

The relative mean-square error $\Delta^{2}$ arising from any mismatch between the predicted and the measured magnitude and phase angle of complex modulus is shown as:

$$
\Delta^{2}=\sum_{i=1}^{N_{1}}\left(\frac{\left|E^{*}\right|_{i}^{\text {pre }}-\left|E^{*}\right|_{i}^{\text {mea }}}{\left|E^{*}\right|_{i}^{\text {mea }}}\right)^{2}+\sum_{i=1}^{N_{2}}\left(\frac{\varphi_{i}^{\text {pre }}-\varphi_{i}^{\text {mea }}}{\varphi_{i}^{\text {mea }}}\right)^{2}
$$

Where $\left|E^{*}\right|_{i}^{\text {pre }}, \varphi_{i}^{\text {pre }},\left|E^{*}\right|_{i}^{\text {mea }}, \varphi_{i}^{\text {mea }}$ are the $i^{\text {th }}$ predicted and measured magnitude and phase angle of complex modulus, respectively. $N_{1}, N_{2}$ are the total number of the observed data of the magnitude and phase angle, respectively.

Table 1 presents the regression parameters of $\delta, \lambda, \kappa, \gamma, \varphi_{\max }, \omega_{0}, \beta$ shown in Equation (1).

Table 1 Parameters of master curves of complex moduli of asphalt mixtures

\begin{tabular}{|c|c|c|c|c|c|c|c|c|c|}
\hline \multirow{2}{*}{ Confinement } & \multicolumn{4}{|c|}{ Dynamic Modulus Master Curve } & \multicolumn{4}{c|}{ Phase Angle Master Curve } \\
\cline { 2 - 10 } & $\delta$ & $\lambda$ & $\kappa$ & $\gamma$ & $R^{2}$ & $\varphi_{\max }$ & $\omega 0$ & $\beta$ & $R^{2}$ \\
\hline 0 & -1.57 & 2.89 & -0.49 & -0.53 & 0.999 & 38.22 & 0.01 & 0.02 & 0.994 \\
\hline $35 \mathrm{kPa}$ & -1.27 & 2.62 & -0.53 & -0.54 & 0.999 & 33.18 & 0.03 & 0.03 & 0.994 \\
\hline $138 \mathrm{kPa}$ & -0.24 & 1.53 & -0.07 & -0.87 & 0.999 & 28.30 & 0.05 & 0.02 & 0.994 \\
\hline $207 \mathrm{kPa}$ & -0.11 & 1.45 & -0.60 & -0.81 & 0.999 & 22.42 & 0.20 & 0.02 & 0.989 \\
\hline
\end{tabular}


Figure 4 gives the master curves of complex moduli of asphalt mixtures at $20^{\circ} \mathrm{C}$ at different confinements. It shows that both dynamic modulus and phase angle are stress-dependent. At the low and intermediate reduced frequencies (e.g., less than $10^{4} \mathrm{~Hz}$ ) the dynamic modulus increases as the increase of the confinement. However, at relatively high reduced frequencies (e.g., greater than $10^{4} \mathrm{~Hz}$ ) there are no obvious differences among the dynamic moduli at different confinements. In terms of phase angle, as the confinement increases the phase angle decreases at relatively low reduced frequencies (e.g., less than $10 \mathrm{~Hz}$ ). However, at the intermediate and high reduced frequencies (e.g., greater than $10 \mathrm{~Hz}$ ), no significant differences were observed among all phase angles under different confinements.

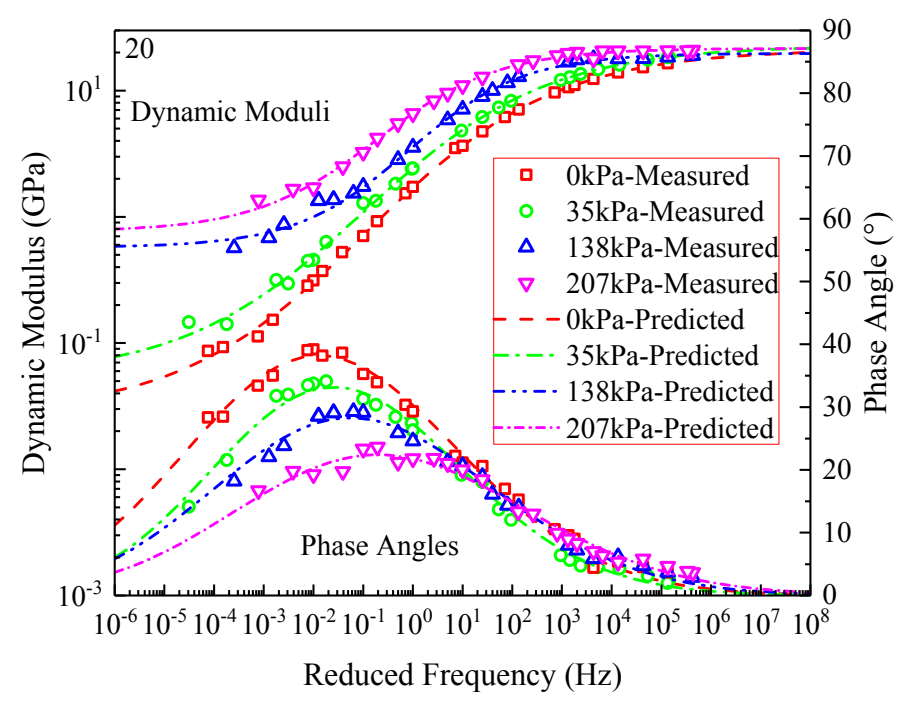

Fig. 4. Complex moduli of asphalt mixtures under different confinements

The fundamental reasons of the above phenomena can be explained by the stress-dependent behaviors of the aggregate skeleton and the viscosity changes of the asphalt binders under different confining pressures $[6,7]$. At a low reduced frequency, the binder of asphalt mixture is relatively less elastic and becomes soft with a low viscosity. Therefore, the aggregates and their skeleton and contact dominate the mechanical behavior of asphalt mixture when a compressive stress is applied. In this case, the stiffness of the binder is so low that the asphalt mixture behaves more like the unbound granular material, which means the aggregate contacts and skeleton in the asphalt mixture are significantly affected by the loading conditions and the material modulus is highly stress-dependent as demonstrated in Figure 4. On the contrary, the 
binder becomes more elastic and harder with a high viscosity at a high-reduced frequency. In this case, both the binder and aggregates will affect the mixtures' response. The effect of the confinement on the aggregate skeleton and contact will much less significant than that a low reduced frequency, due to the higher stiffness of the binder and the elevated adhesion at the binder-aggregate interfaces. The asphalt mixture behaves as a linear elastic solid with much less effect of the stress conditions on the material properties.

\subsection{Stress-dependent Long-term Equilibrium Modulus}

Apart from the complex modulus, the relaxation modulus is also commonly used as a material property to characterize the material viscoelastic behavior. In this section, Prony series representation shown in Equation (4) is used to model the relaxation modulus of asphalt mixture.

$$
E(t)=E_{\infty}+\sum_{i=1}^{N} E_{i} \exp \left(-\frac{t}{\rho_{i}}\right)
$$

Where $E_{\infty}$ is long-term equilibrium modulus. Physically $E_{\infty}$ is the dynamic modulus when the reduced frequency becomes zero and mathematically $E_{\infty}$ equals to $10^{\delta}$. $E_{i}$ is the modulus in the $i^{\text {th }}$ Maxwell component. $\rho_{i}$ is relaxation time in the $i^{\text {th }}$ Maxwell component. $t$ is loading time. In addition, $N$ is total number of Maxwell components.

Asphalt mixture manifests the similar stress-dependent modulus behavior as the unbound granular materials in a low frequency, as demonstrated in Figure 4. Therefore, it is proposed to employ the Uzan Model to characterize the stress-dependence of $E_{\infty}$ of the asphalt mixtures:

$$
E_{\infty}=k_{1} P_{a}\left(\frac{I_{1}}{P_{a}}\right)^{k_{2}}\left(\frac{\tau_{o c t}}{P_{a}}\right)^{k_{3}}
$$

Where $P_{a}$ is atmospheric pressure. $I_{1}$ is bulk stress. $\tau_{\text {oct }}$ is octahedral stress. $k_{1}, k_{2}$, and $k_{3}$ are model coefficients. It is noted that the values of $I_{1}$ and $\tau_{\text {oct }}$ in Equation (5) should be calculated at the infinite loading time or the infinitesimal reduced frequency according to the definition of $E_{\infty}$ in Equation (4). Therefore, a master curve method was proposed herein to obtain the deviatoric stresses at the infinitesimal reduced frequency under different confining pressures. The deviatoric stresses recorded during the complex modulus tests are shown in Figure 5. 

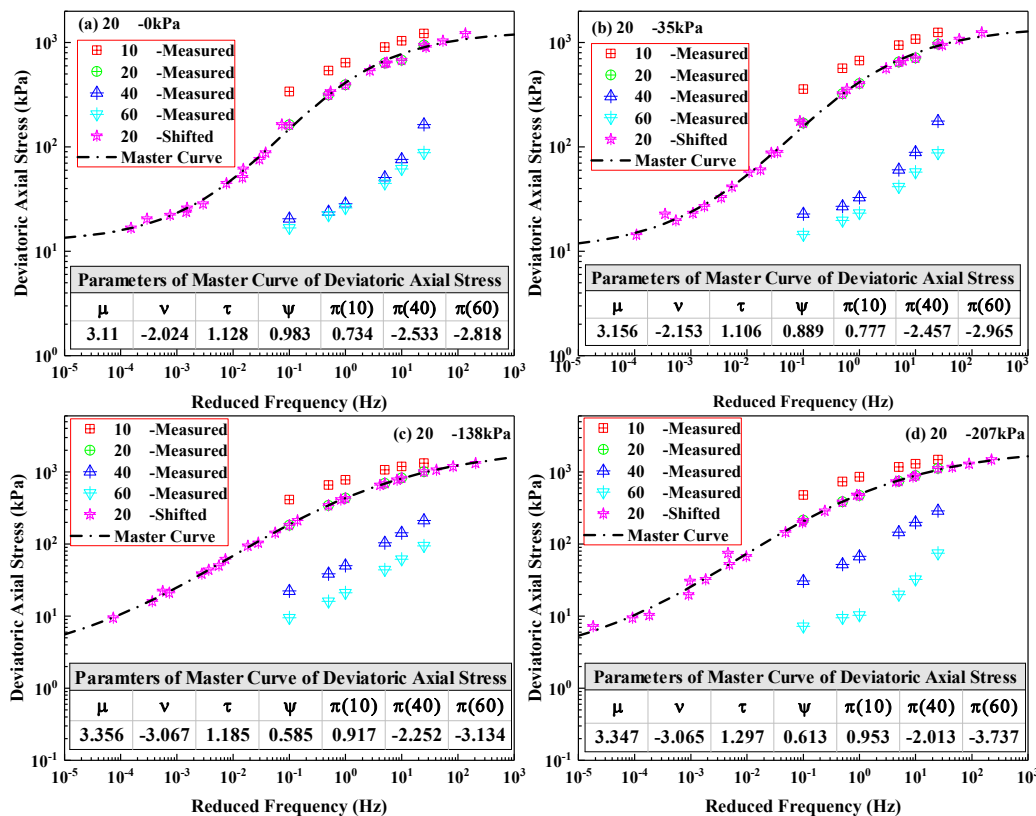

\section{Fig. 5. Deviatoric stresses recorded in the tests and their master curves at $20^{\circ} \mathrm{C}$}

Figure 5 shows that the deviatoric stresses recorded during the complex modulus tests are frequency (or time) and temperature dependent. The concept of master curve used herein is to estimate the deviatoric stresses at the infinitesimal frequency. Like the dynamic modulus model shown in Equation (1), the model for deviatoric stress master curve can be proposed as

$$
\log _{10} \sigma_{d}=\mu+\frac{v}{1+\exp \left(\tau+\psi \log _{10} \omega_{R}^{\sigma_{d}}\right)}
$$

Where $\sigma_{d}$ is the deviatoric stress. $\mu$ is the maximum value of $\log _{10} \sigma_{d .}-\mathrm{v}$ is the span of $\log _{10} \sigma_{d}$. $\tau$ and $\Psi$ are the shape parameters for the proposed master curve. $\omega_{R}^{\sigma_{d}}$ is the reduced frequency used to describe the master curve of $\sigma_{d}$, which can be obtained by:

$$
\omega_{R}^{\sigma_{d}}=\pi(T) \omega
$$

Where, $\pi(T)$ is a time-temperature shift factor for the master curve of $\sigma_{d}, \omega$ is loading frequency, and $T$ is temperature.

Figure 5 demonstrates that the time-temperature superposition principle and the master curve models are applicable to the deviatoric stress in the complex modulus test. This is fundamentally because the complex modulus tests are conducted in a strain-controlled mode. 
According to the definition of dynamic modulus, the changes of dynamic modulus remains the same as that of the deviatoric stress when the strains are controlled unchanged with load cycles in each test. Therefore, the time-temperature superposition can be used to construct the master curve for not only the dynamic modulus but also the deviatoric stresses.

The deviatoric stress at the infinitesimal reduced frequency can be calculated by

$$
\sigma_{d, \infty}=10^{\mu+v}
$$

Where, $\sigma_{d, \infty}$ is the deviatoric stress at the infinitesimal reduced frequency. $\mu$ and $\pi$ are explained in Equation (6). Based on equation (8), the values of $\sigma_{d, \infty}$ are determined as $12.19 \mathrm{kPa}, 10.08$ $\mathrm{kPa}, 1.95 \mathrm{kPa}$ and $1.91 \mathrm{kPa}$ at the confinements of $0,35 \mathrm{kPa}, 138 \mathrm{kPa}$, and $207 \mathrm{kPa}$ respectively. After the determinations of $\sigma_{d, \infty}$, hydraulic bulk stress $I_{1}$ and octahedral shear stress $\tau_{\text {oct }}$ can be calculated, and Equation (5) allows for the use of Microsoft Excel spreadsheets and solver function to attain the regression values of $k_{1}, k_{2}$, and $k_{3}$ with the known values of $E_{\infty}$. The values of $k_{1}, k_{2}$, and $k_{3}$ are $9.406,0.207$, and -1.315 , respectively, with a $\mathrm{R}^{2}$ of 0.992 . The positive $k_{2}$ and negative $k_{3}$ indicate that a hydraulic bulk stress will strengthen $E_{\infty}$ while the octahedral stress will weaken $E_{\infty}$, which is consistent with the resilient modulus behavior of the unbound granular aggregate materials. The high $R^{2}$ value and the consistency with the unbound materials demonstrate that the commonly used Uzan model can characterize the stress-dependent behavior of $E_{\infty}$ of asphalt mixtures. The Uzan model coefficient can be determined effectively by using the proposed deviatoric stress master curves that gives the stress states over the fullreduced frequency range.

\subsection{Stress-dependent Modulus in $i^{\text {th }}$ Maxwell Component}

After determining the stress dependence of the long-term equilibrium modulus, the focus of this section is to analyze the modulus and relaxation time in the Maxwell components in Prony Series model of relaxation modulus. Thus, the Maxwell component coefficients $\left(E_{i}\right.$ and $\rho_{i}$ in Equation (4)) will be determined and their stress dependence will be discussed. Different methods [14-17] are available to obtain the Maxwell component coefficients based on the master curves of complex modulus of polymer. However, it's demonstrated that the above methods may not applicable for asphalt mixture due to the inclusion of air voids, aggregates, 
adhesion between bitumen and aggregates, and test environment [25]. Therefore, a simple and robust method will be proposed in the first part of this section followed by a discussion of the stress dependence of relaxation spectrums of asphalt mixtures at different confining pressures.

\subsubsection{Determination of the Maxwell component coefficients}

One of the most common techniques used to calculate the relaxation modulus is collocation method proposed by Schapery [26]. Figure 6 gives the results of relaxation spectrum $\left(E_{i} \sim \rho_{i}\right)$ of asphalt mixtures, which is calculated with Schapery's collocation method.
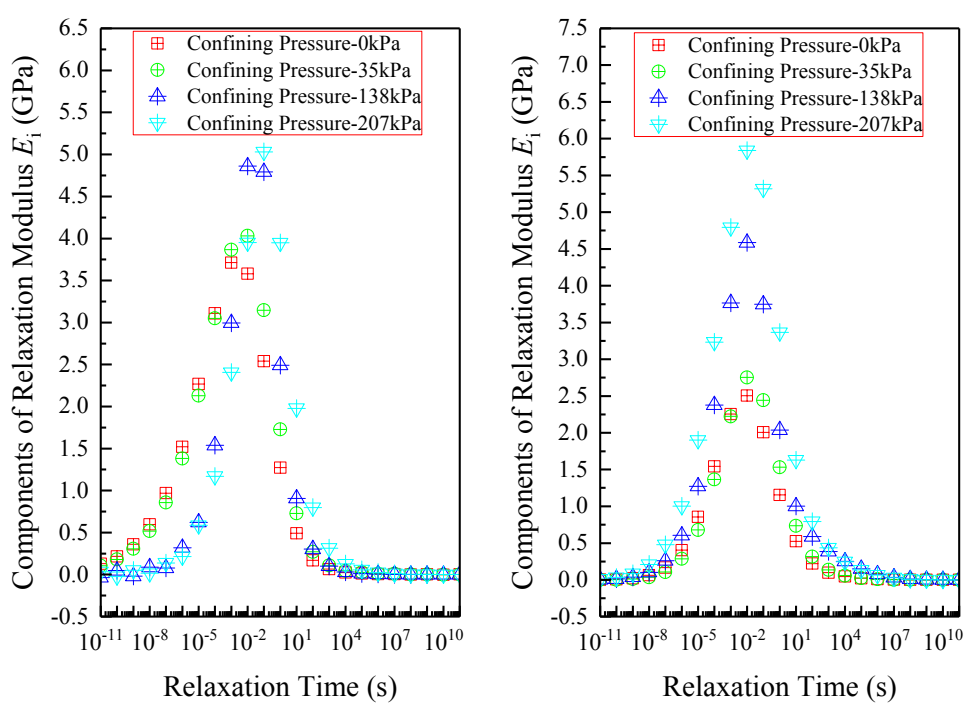

Fig. 6 Relaxation spectrum of asphalt mixture at $20^{\circ} \mathrm{C}$ and different confining pressures

Figure 6 shows that the relaxation spectrums obtained from storage and loss moduli are different for the same asphalt mixture. This implies that the collocation method used herein may not reliable. Actually, $E_{i}$ obtained from the loss moduli is a little more reasonable than the one calculated from the storage moduli. The reason is that there are one (for 0, 35 and $207 \mathrm{kPa}$ ) or two negative (for $138 \mathrm{kPa}$ ) $E_{i}$ generated when the measured storage moduli are used. However, no negative $E_{i}$ was obtained when the loss moduli are used. To obtain accurate enough $E_{i}$, a two-step procedure (improved collocation method) is proposed. The first step is to estimate the initial values of $E_{i}$ with Schapery's collocation method. The second step is to obtain accurate $E_{i}$ by a generalized reduced gradient nonlinear least-squares regression method and the initial values of $E_{i}$. The key in the second step is to choose a proper regression minimization objective (RMO). Therefore, the below four candidate regression objectives are proposed: 


$$
\begin{aligned}
& \Delta_{1}^{2}=\sum_{i=1}^{N}\left\{\left[E_{\text {mea }}^{\prime}\left(\omega_{i}\right)-E_{\text {pre }}^{\prime}\left(\omega_{i}\right)\right]^{2}+\left[E_{\text {mea }}^{\prime \prime}\left(\omega_{i}\right)-E_{\text {pre }}^{\prime \prime}\left(\omega_{i}\right)\right]^{2}\right\} \\
& \Delta_{2}^{2}=\sum_{i=1}^{N}\left\{\left[\frac{E_{\text {mea }}^{\prime}\left(\omega_{i}\right)-E_{\text {pre }}^{\prime}\left(\omega_{i}\right)}{E_{\text {mea }}^{\prime}\left(\omega_{i}\right)}\right]^{2}+\left[\frac{E_{\text {mea }}^{\prime \prime}\left(\omega_{i}\right)-E_{\text {pre }}^{\prime \prime}\left(\omega_{i}\right)}{E_{\text {mea }}^{\prime \prime}\left(\omega_{i}\right)}\right]^{2}\right\} \\
& \Delta_{3}^{2}=\sum_{i=1}^{N}\left\{\left[\log \left(E_{\text {mea }}^{\prime}\left(\omega_{i}\right)\right)-\log \left(E_{\text {pre }}^{\prime}\left(\omega_{i}\right)\right)\right]^{2}+\left[\log \left(E_{\text {mea }}^{\prime \prime}\left(\omega_{i}\right)\right)-\log \left(E_{\text {pre }}^{\prime \prime}\left(\omega_{i}\right)\right)\right]^{2}\right\} \\
& \Delta_{4}^{2}=\sum_{i=1}^{N}\left\{\left[\frac{\log \left(E_{\text {mea }}^{\prime}\left(\omega_{i}\right)\right)-\log \left(E_{\text {pre }}^{\prime}\left(\omega_{i}\right)\right)}{\log \left(E_{\text {mea }}^{\prime}\left(\omega_{i}\right)\right)}\right]^{2}+\left[\frac{\log \left(E_{\text {mea }}^{\prime \prime}\left(\omega_{i}\right)\right)-\log \left(E_{\text {pre }}^{\prime \prime}\left(\omega_{i}\right)\right)}{\log \left(E_{\text {mea }}^{\prime \prime}\left(\omega_{i}\right)\right)}\right]^{2}\right\}
\end{aligned}
$$

To determine which of the four RMOs give the best accuracy of the regression, four important criteria are employed. They are highest value of $\mathrm{R}^{2}$, least value of Error calculated by Equation (10), least difference between the Errors calculated from the measured storage and loss moduli, and least difference for $E_{i}$ calculated from the storage and loss modulus.

$$
\text { Error }=\sum_{i=1}^{N}\left\{\left[\frac{E_{\text {mea }}^{\prime}\left(\omega_{i}\right)-E_{\text {pre }}^{\prime}\left(\omega_{i}\right)}{E_{\text {mea }}^{\prime}\left(\omega_{i}\right)}\right]^{2}+\left[\frac{E_{\text {mea }}^{\prime \prime}\left(\omega_{i}\right)-E_{\text {pre }}^{\prime \prime}\left(\omega_{i}\right)}{E_{\text {mea }}^{\prime \prime}\left(\omega_{i}\right)}\right]^{2}\right\}
$$

Where $E_{\text {mea }}^{\prime}\left(\omega_{i}\right)$ and $E_{\text {pre }}^{\prime}\left(\omega_{i}\right)$ are the $i^{\text {th }}$ measured and predicted storage modulus; $E_{\text {mea }}^{\prime \prime}\left(\omega_{i}\right)$ and $E_{\text {pre }}^{\prime \prime}\left(\omega_{i}\right)$ are the $i^{\text {th }}$ measured and predicted loss modulus, respectively.

Figures 7 presents the results of RMOs, where $\Delta_{i-\text { Storge }}^{2}$ and $\Delta_{i-\text { Loss }}^{2}$ are the RMOs when the measured storage and loss moduli were used in the collocation method, respectively. Moreover, $\Delta_{i-i}^{2}$ implies the difference of Errors.

Figures 7 shows that $\Delta_{3}^{2}$ shown in Equation (9) is the best one to calculate $E_{i}$ because it yields a relatively larger value of $\mathrm{R}^{2}$, a relatively smaller value of Error calculated by Equation (10), and the least difference of the Errors calculated from the measured storage and loss moduli. In addition, only $\Delta_{3}^{2}$ can yield the identical $E_{i}$ when storage and loss moduli were used, respectively. In addition, Figure 8 presents the measured and predicted (RMO $\left.\Delta_{3}^{2}\right)$ storage and loss moduli with the improved collocation method.

In summary, the key points of estimating $E_{i}$ can be summarized as: 1) it is suggested to use the measured loss moduli rather than storage moduli to calculate $E_{i}$ if the collocation method is 
utilized; and 2) the RMO $\Delta_{3}^{2}$ in Equation (9) should be used as the regression criterion during the coefficient determination.
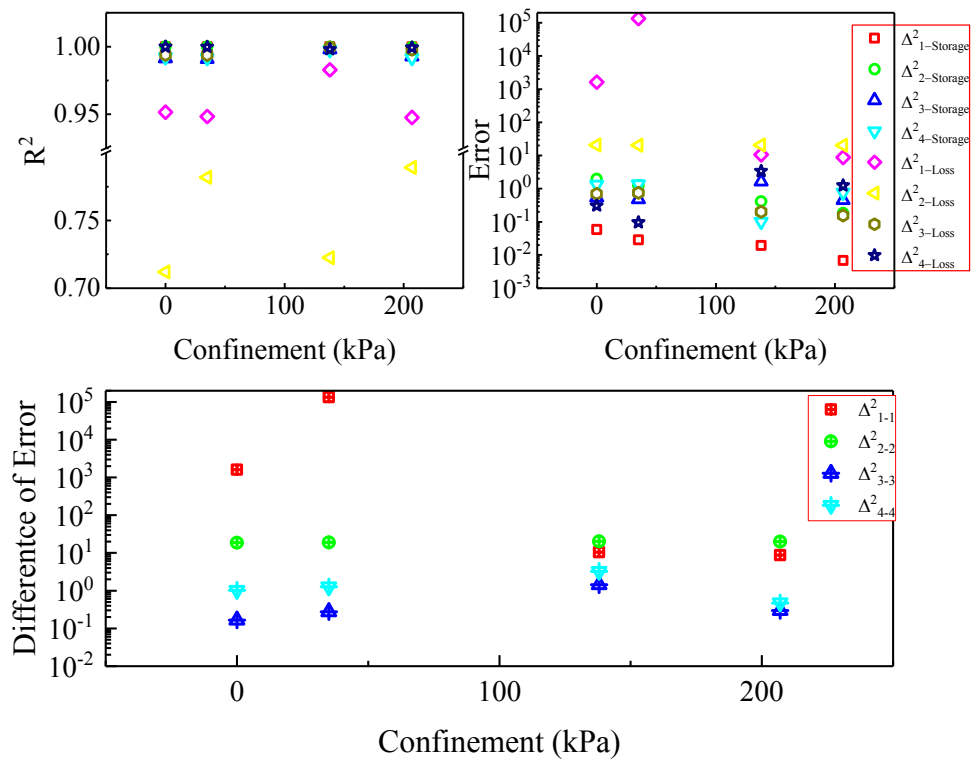

Fig. 7. $\mathbf{R}^{2}$, Error, and difference of Errors of asphalt mixtures at different confinements
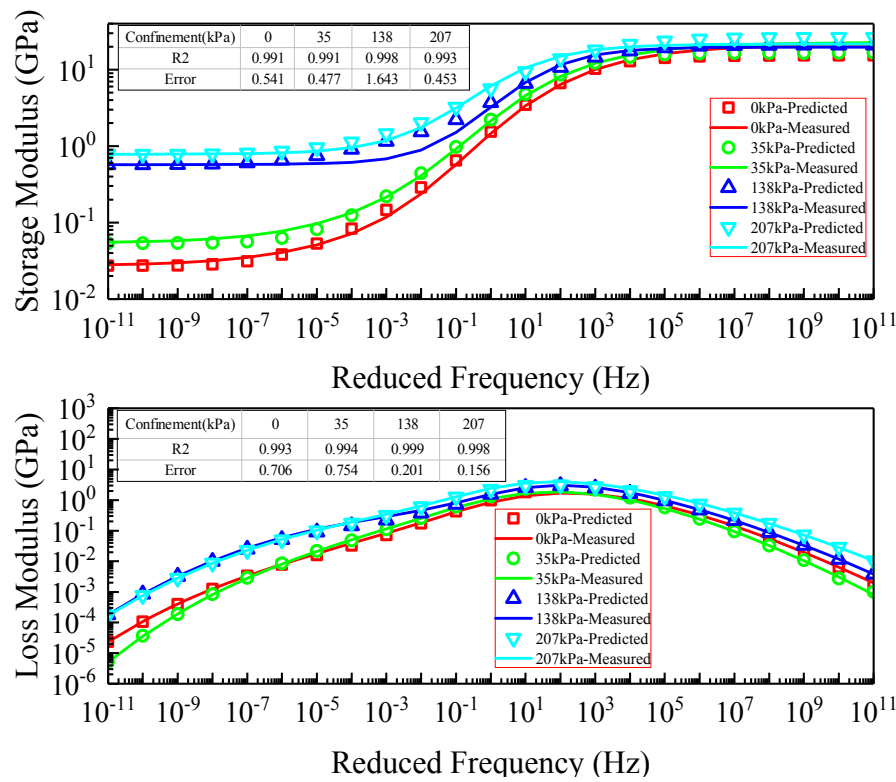

Fig. 8. Measured and predicted storage and loss moduli of asphalt mixtures

\subsubsection{Stress Dependence of Relaxation Spectrum}

The mechanical analogs of Prony series model can be represented by a solid-like generalized Maxwell model, and the long-term equilibrium modulus can be regarded as a special component of relaxation modulus with relaxation time equaling to $\infty$. Now that the long-term equilibrium modulus $E_{\infty}$ can be fitted with Uzan model, it is spontaneous to utilize the same regression 
function to model the stress dependence of $E_{i}$. Considering that the master curves of deviatoric stresses have been obtained in Figures 5, we can use the similar procedure as dealing with $E_{\infty}$ to analyze the stress dependence of $E_{i}$. However, because there are many Maxwell element branches in the generalized Maxwell model, and if we investigate the stress dependence of $E_{i}$ one by one, it is a time-consuming and labor-intensive process. Moreover, this method will generate too many coefficients to be determined, which will make the regression results unreliable. Therefore, we propose an alternative way to deal with the stress dependence of $E_{i}$. The dotted data in Figure 9 show the relaxation spectrum based on the predicted storage and loss moduli of asphalt mixtures at different confinements.

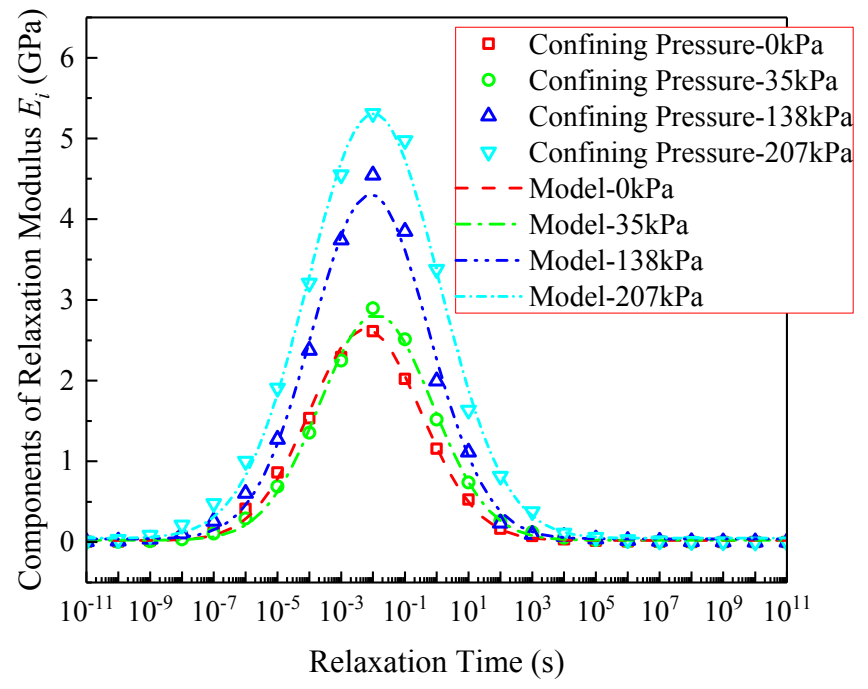

Fig. 9. Relaxation spectrum of asphalt mixtures at $20^{\circ} \mathrm{C}$

It is found that $E_{i}$ increases with the increase of confining pressure. Physically, only the size of relaxation spectrum is stress dependent, and the flatness of shape and the position of the maximum of $E_{i}$ are stress independent. Hence, each relaxation spectrum can be modeled by Gaussian function as in Equation (11) and shown as the dashed lines in Figure 8:

$$
E\left(t_{R i}, \sigma\right)=\frac{a(\sigma)}{\sqrt{\pi}} \exp \left[-b^{2}\left(\log t_{R i}-\log c\right)^{2}\right]
$$

Where, $t_{R i}$ is the $i^{\text {th }}$ relaxation time (second). $\sigma$ is stress $(\mathrm{GPa}) . a(\sigma), b$, and $c$ are fitting parameters with a dimension of GPa, 1 , and second, respectively. The parameter of $a(\sigma)$ is stress dependent. Parameters of $a, b$, and $c$ in Equation (11) can be obtained and shown in Table 2. 
Table 2 Coefficients of Gaussian function of the relaxation spectrum of asphalt mixtures

\begin{tabular}{|c|c|c|c|c|}
\hline Confinements $(\mathrm{kPa})$ & 0 & 35 & 138 & 207 \\
\hline$a(\mathrm{GPa})$ & 4.502 & 4.997 & 7.854 & 9.385 \\
\hline$b$ & 0.379 & 0.379 & 0.379 & 0.379 \\
\hline$c$ (second) & 0.009 & 0.009 & 0.009 & 0.009 \\
\hline $\mathrm{R}^{2}$ & 0.994 & 0.996 & 0.996 & 0.996 \\
\hline
\end{tabular}

As seen from Table 2 and Figure 9, Equation (11) delivers a very good approximation of $E_{i}$ of asphalt mixtures at different confining pressures. A regression equation like Equation (5) is utilized to characterize the stress dependence of parameter $a$ :

$$
a(\sigma)=l_{1} P_{a}\left(\frac{I_{1}}{P_{a}}\right)^{l_{2}}\left(\frac{\tau_{o c t}}{P_{a}}\right)^{l_{3}}
$$

Where $l_{1}, l_{2}$, and $l_{3}$ are the regression parameters. The values of $l_{1}, l_{2}$, and $l_{3}$ are 215.711, 0.057, and 3.240, respectively, with $\mathrm{R}^{2}$ equaling to 0.997 .

Another eight different lab-fabricated asphalt mixtures were fabricated and tested to determine $E_{\infty}$ and $E_{i}$ of relaxation modulus using the improved collocation method proposed in this paper. Uzan model mentioned herein can also be utilized to calculate $E_{\infty}$ and $E_{i}$ of two of them accurately (another six mixtures are only tested in one confining pressure), and the model coefficients $\left(k_{1}, k_{2}, k_{3}, l_{1}, l_{2}\right.$, and $\left.l_{3}\right)$ are different because of the difference in the mixture components. We did not include the above information in this paper due to that this paper is focused on the model development of the stress dependent modulus including only a preliminary validation on one mixture. A comprehensive validation on the eight mixtures will be presented in the following publications. It would become a lengthy paper if put the two significant work in one paper which will beyond the journal word limit.

\section{Summary and Conclusions}

This paper investigated the stress dependence of asphalt mixtures by strain-controlled complex modulus tests at different confining pressures. The master curves of dynamic modulus and phase angle at different confining pressures were firstly constructed using sigmoidal model and beta model, respectively. Then the stress dependence of the long-term equilibrium modulus of 
asphalt mixture based on Prony series model was modeled by Uzan model, in which the concept of deviatoric stress master curves are firstly proposed to obtain the stress states of asphalt mixtures over the full loading frequency. After that, the Maxwell components of the relaxation modulus were determined by an improved collocation method in which two critical criteria were proposed to regress reliable and accurate model coefficients. Finally, the stress dependence of the relaxation modulus was quantified by the relaxation spectrum. The main findings and conclusions of this paper are summarized as follows:

(1) For selected dense-graded asphalt mixtures, the dynamic modulus increases, and phase angle decreases with an increasing confining pressures at relatively low reduced loading frequency (e.g., less than $10 \mathrm{~Hz}$ ). However, the stress dependence is insignificant in a relatively high reduced loading frequency (e.g., greater than $10^{4} \mathrm{~Hz}$ ). This phenomenon can be ascribed to the stress-dependent behaviors of the aggregate skeleton and the viscosity changes of the asphalt binders at different confining pressures.

(2) The commonly used Uzan model is demonstrated to be able to characterize the stressdependent behavior of the long-term equilibrium modulus of the relaxation modulus for the asphalt mixtures. The Uzan model coefficient can be determined effectively by using the proposed deviatoric stress master curves that give the stress states over the full-reduced frequency range.

(3) To obtain the reliable and accurate $E_{i}$, it is recommended that: a) the measured loss moduli rather than the storage moduli should be used if the collocation method is utilized; and b) the log-scaled least squared regression minimization objective $\Delta_{3}^{2}$ in Equation (9) should be used as the regression criterion during the coefficient determination.

(4) The relaxation spectrums of asphalt mixtures can be modeled by Gaussian function, where the size of the relaxation spectrum increases but the shape and position of the relaxation spectrum remain unchanged when the confining pressure increases. Furthermore, the size parameter $a$ in the Gaussian function can be modeled by Uzan model to quantify the stressdependence of the relaxation modulus of asphalt mixtures.

\section{Acknowledgements}


The authors would like to acknowledge the financial support from the National Nature Science Foundation of China (Grant No. 51408173), Marie Skłodowska-Curie Individual Fellowships under EU's H2020 programme (Grant No. 789551), China Postdoctoral Science Foundation funded Project (Grant No. 2015M571928), and Fundamental Research Funds for the Central Universities in China (Grant Nos. JZ2018HGTB0258).

\section{References}

[1] L. Tashman, M. Elangovan, Dynamic Modulus of HMA and Its Relationship to Actual and Predicted Field Performance Using MEPDG, Journal of Performance of Constructed Facilities 27(3) (2013) 334-345.

[2] S. Yang, A. Braham, S. Underwood, A. Hanz, G. Reinke, Correlating field performance to laboratory dynamic modulus from indirect tension and torsion bar, Road Materials and Pavement Design 18 (2017) 104-127.

[3] Y.R. Kim, Y. Seo, M. King, M. Momen, Trb, Dynamic modulus testing of asphalt concrete in indirect tension mode, Bituminous Paving Mixtures 20042004, pp. 163-173.

[4] T.K. Pellinen, M.W. Witczak, R.F. Bonaquist, Asphalt mix master curve construction using sigmoidal fitting function with non-linear least squares optimization technique, In: Proceedings of 15th ASCE engineering mechanics conference, Columbia University, New York; 2002.

[5] R. Luo, H. Liu, Improving the Accuracy of Dynamic Modulus Master Curves of Asphalt Mixtures Constructed Using Uniaxial Compressive Creep Tests, Journal of Materials in Civil Engineering 29(7) (2017).

[6] A. Lacroix, B.S. Underwood, Y.R. Kim, Reduced Testing Protocol for Measuring the Confined Dynamic Modulus of Asphalt Mixtures, Transportation Research Record (2210) (2011) 20-29.

[7] W.A.-E. Zeiada, K.E. Kaloush, K.P. Biligiri, J.X. Reed, J.J. Stempihar, Significance of Confined Dynamic Modulus Laboratory Testing for Asphalt Concrete Conventional, GapGraded, and Open-Graded Mixtures, Transportation Research Record (2210) (2011) 9-19. 
[8] A. Sotil, K.E. Kaloush, M.W. Witczak, Trb, Reduced confined dynamic modulus testing protocol for asphalt mixtures, Bituminous Paving Mixtures 20042004, pp. 153-162.

[9] T. Pellinen, M. Witczak, Stress dependent master curve construction for dynamic (complex) modulus (with discussion), Journal of the Association of Asphalt Paving Technologists 71 (2002).

[10] M. Witczak, J. Uzan, The universal airport pavement design system, Report I of V: granular material characterization, Department of Civil Engineering, University of Maryland, College Park, MD (1988).

[11] R. Lytton, Foundations and pavements on unsaturated soils, Proceedings of the first international conference on unsaturated soils/unsat'95/Paris/France/6-8 September 1995. Volume 3, 1996.

[12] P.V. Lade, R.B. Nelson, Modelling the elastic behaviour of granular materials, International journal for numerical and analytical methods in geomechanics 11(5) (1987) 521-542.

[13] A. Collop, A. Scarpas, C. Kasbergen, A. de Bondt, Development and finite element implementation of stress-dependent elastoviscoplastic constitutive model with damage for asphalt, Transportation Research Record: Journal of the Transportation Research Board (1832) (2003) 96-104.

[14] M. Baumgaertel, H. Winter, Determination of discrete relaxation and retardation time spectra from dynamic mechanical data, Rheologica Acta 28(6) (1989) 511-519.

[15] I. Emri, N. Tschoegl, Generating line spectra from experimental responses. Part I: Relaxation modulus and creep compliance, Rheologica Acta 32(3) (1993) 311-322.

[16] T.L. Cost, E.B. Becker, A multidata method of approximate Laplace transform inversion, International journal for numerical methods in engineering 2(2) (1970) 207-219.

[17] R. Schapery, A simple collocation method for fitting viscoelastic models to experimental data, (1962).

[18] N.W. Tschoegl, The phenomenological theory of linear viscoelastic behavior: an introduction, Springer Science \& Business Media2012. 
[19] Y. Zhang, F. Gu, X. Luo, B. Birgisson, R.L. Lytton, Modeling Stress-Dependent Anisotropic Elastoplastic Unbound Granular Base in Flexible Pavements, Transportation Research Record (2018) 0361198118758318.

[20] T. Officials, Standard specifications for transportation materials and methods of sampling and testing, AASHTO2011.

[21] Y. Zhang, R. Luo, R.L. Lytton, Anisotropic Viscoelastic Properties of Undamaged Asphalt Mixtures, Journal of Transportation Engineering-Asce 138(1) (2012) 75-89.

[22] Y. Zhang, R. Luo, R.L. Lytton, Characterizing Permanent Deformation and Fracture of Asphalt Mixtures by Using Compressive Dynamic Modulus Tests, Journal of Materials in Civil Engineering 24(7) (2012) 898-906.

[23] B. Rahimzadeh, Linear and non-linear viscoelastic behaviour of binders and asphalts, University of Nottingham, 2002.

[24] Y. Zhang, B. Birgisson, R.L. Lytton, Weak Form Equation-Based Finite-Element Modeling of Viscoelastic Asphalt Mixtures, Journal of Materials in Civil Engineering 28(2) (2015) 04015115.

[25] L. Li, W. Li, H. Wang, J. Zhao, Z. Wang, M. Dong, D. Han, Investigation of Prony series model related asphalt mixture properties under different confining pressures, Constr Build Mater 166 (2018).

[26] R. Schapery, Two simple approximate methods of Laplace transform inversion for viscoelastic stress analysis, (1961). 